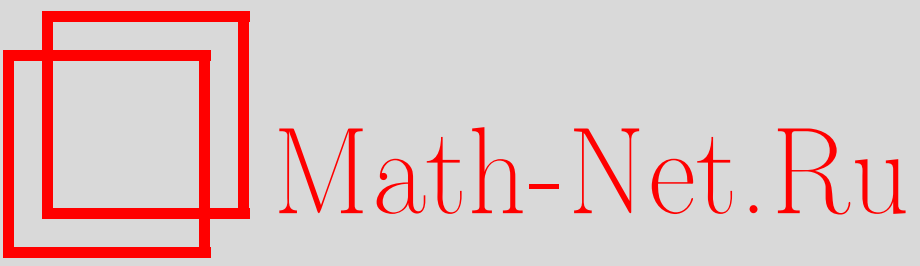

Я. Б. Воробец, Асимптотика спектра оператора Лапласа-Бельтрами на торах с лиувиллевыми и инфралиувиллевыми метриками, УМН, 1997, том 52, выпуск 2, 163-164

DOI: https://doi.org/10.4213/rm832

Использование Общероссийского математического портала Math-Net.Ru подразумевает, что вы прочитали и согласны с пользовательским соглашением

http://www. mathnet.ru/rus/agreement

Параметры загрузки:

IP: 54.198 .64 .247

26 апреля 2023 г., 14:10:30 


\title{
АСИМПТОТИКА СПЕКТРА ОПЕРАТОРА ЛАПЛАСА-БЕЛЬТРАМИ НА ТОРАХ С ЛИУВИЛЛЕВЫМИ И ИНФРАЛИУВИЛЛЕВЫМИ МЕТРИКАМИ
}

\author{
Я.Б. ВоРОБЕЦ
}

Рассмотрим двумерньй тор $T_{a, b}=\mathbb{R}^{2} /(a \mathbb{Z} \oplus b \mathbb{Z})(a, b>0)$, с естественными циклическими координатами $x_{1}, x_{2}$. Риманова метрика на торе $T_{a, b}$ вида

$$
d s^{2}=\left(U_{1}\left(x_{1}\right)-U_{2}\left(x_{2}\right)\right)\left(d x_{1}^{2}+d x_{2}^{2}\right)
$$

называется метрикой Лиув илля. Здесь $U_{1}, U_{2}$ - непрерывные периодические функции на $\mathbb{R}$ с периодами $a$ и $b$, соответственно, причем $\min _{x \in \mathbb{R}} U_{1}(x)>\max _{x \in \mathbb{R}} U_{2}(x)$. Частным случаем таких метрик являются метрики вращения на торе. Они получаются из формулы (1) при $U_{2} \equiv 0$.

ОПреДЕЛЕниЕ 1. Лиувиллев а метрика на двумерном торе - это риманова метрика, которая в каких-либо циклических координатах $x_{1}, x_{2}$ на торе представима в виде $(1)$.

Пусть лиувиллева метрика вида (1) на торе $T_{a, b}$ инвариантна относительно некоторой конечной группы $G$ сдвигов тора. Тогда она факторизуется до римановой метрики на торе $T_{a, b} / G$. Фактор-метрика локально представима в виде (1). Если группа $G$ не разлагается в прямую сумму двух подгрупп $G_{1}$ и $G_{2}$, где $G_{1}$ содержит сдвиги только вдоль $x_{1}$, а $G_{2}$ - сдвиги только вдоль $x_{2}$, то глобалшного представления вида $(1)$, вообще говоря, нет, т.е. метрика не лиувиллева. А.М. Стёпин предложил называть такие метрики инфралиувиллевыми.

ОПредЕлЕниЕ 2. Риманова метрика на торе назьвается инфралиувиллевой, если она не лиувиллева, но конечнолистно накрьвается лиувиллевой метрикой.

В настоящей работе рассматривается оператор Лапласа-Бельтрами на торах с лиувиллевыми и инфралиувиллевыми метриками. В лиувиллевых координатах (т.е. координатах, в которых метрика имеет вид (1)) он задается формулой

$$
\Delta=\left(U_{1}\left(x_{1}\right)-U_{2}\left(x_{2}\right)\right)^{-1}\left(\frac{\partial^{2}}{\partial x_{1}^{2}}+\frac{\partial^{2}}{\partial x_{2}^{2}}\right) .
$$

Оператор $\Delta$ является самосопряженным неположительным оператором с дискретным спектром в гилшбертовом пространстве $L_{2}(T, \mu)$, где $T$ - рассматриваемьй тор, $\mu$ - элемент площади соответствующей метрики.

Оператор Лапласа-Бельтрами можно рассматривать как квантовый гамильтониан, соответствующий классическому гамилтониану геодезического потока на $T$. Геодезический поток имеет дополнительньй интеграл, квадратичный по импульсам (согласно [1], [2], только рассматриваемые метрики обладают таким свойством). Это находит свое отражение в том, что спектральная задача для оператора $\Delta$ в случае лиувиллевой метрики оказьвается задачей с разделяющимися переменными (а именно, переменные разделяются в лиувиллевых координатах). В случае инфралиувиллевой метрики переменные не разделяются, однако наличие лиувиллевого накрытия позволяет свести спектральную задачу к задаче (точнее, к нескольким независимым задачам) с разделяющимися переменными.

Для произвольного $x>0$ обозначим через $N(x)$ число собственных значений (с учетом кратности) оператора $-\Delta$, не превосходящих $x$. Согласно формуле Вейля, $N(x)=\frac{\operatorname{Area~} T}{4 \pi} x+o(x)$ при $x \rightarrow \infty$. Теорема 1 , приводимая ниже, дает для типичной лиувиллевой или инфралиувиллевой метрики более точную оценку остаточного члена в этой асимптотической формуле. Чтобы придать строгий смысл понятию типичности, введем пространства лиувиллевых и инфралиувиллевых метрик. Через $\mathscr{L}^{r}(a, b)$ обозначим множество метрик вида (1) на торе $T_{a, b}$, для которых функции $U_{1}$ и $U_{2}$ непрерьвно диффференцируемы $r$ раз $(0 \leqslant r \leqslant \infty)$. Иньми словами,

Работа выполнена при финансовой поддержке Российского фонда фундаментальных исследований (грант № 96-01-00713). 
это пространство лиувиллевых метрик гладкости $C^{r}$ на торе, имеющих фиксированные лиувиллевы координаты. Каждая метрика из $\mathscr{L}^{r}(a, b)$ задается парой функций $U_{1}, U_{2} \in C^{r}(\mathbb{R})$, что позволяет перенести на метрики топологию из $C^{r}(\mathbb{R}) \times C^{r}(\mathbb{R})$. Далее, пусть $G$ - произвольная конечная группа сдвигов тора $T_{a, b}$, которая не разлагается в прямую сумму групп, содержащих сдвиги только вдоль одной координаты. Через $\mathscr{L}_{\text {infra }}^{r}(a, b ; G)$ обозначим пространство метрик на торе $T_{a, b} / G$, которые являются проекциями метрик из $\mathscr{L}^{r}(a, b)$, с топологией, унаследованной от $\mathscr{L}^{r}(a, b)$. Это пространство инфралиувиллевых метрик гладкости $C^{r}$ на торе, для которых фиксируется лиувиллево накрытие, к тому же на накрывающем торе зафиксированы лиувиллевы координаты. Заметим, что пространство $\mathscr{L}_{\text {infra }}^{r}(a, b ; G)$ содержит и лиувиллевы метрики, однако последние нигде не плотны в нем.

Tеорема 1. При $r \geqslant 5$ в пространствах $\mathscr{L}^{r}(a, b)$ и $\mathscr{L}_{\text {infra }}^{r}(a, b ; G)$ найдутся остаточные подмножества, для метрик из которых спектральная функция оператора Лапласа-Бельтрами имеет вид

$$
N(x)=\frac{\text { Area } T}{4 \pi} x+x^{1 / 4} \theta\left(x^{1 / 2}\right)
$$

где $\theta$ - почти-периодическая по Безиковичу функция из пространства $B^{1}[0, \infty)$.

Отметим, что, ввиду почти-периодичности, функция $\theta$ является ограниченной "в среднем". В то же время для некоторых значений аргумента она может принимать (и в типичных случаях принимает) сколь угодно болшшие по модулю значения, как положительные, так и отрицательные. Теорема 1 ничего не утверждает о порядке таких "болшших уклонений”.

Теорема 1 обобщает аналогичное утверждение Д. В. Косыгина, А. А. Минасова и Я. Г. Синая [3], доказанное ими для одного специального класса лиувиллевых метрик, а именно, метрик из пространства $\mathscr{L}^{r}(1,1)$, для которых соответствующие функции $U_{1}, U_{2}$ имеют на периоде всего две невырожденные критические точки - один максимум и один минимум.

Основные отличия в доказательстве теоремы 1 по сравнению с [3] относятся к выводу асимптотических формул для собственных значений оператора Лапласа-Белштрами (правил квантования). Остановимся на этом чуть подробне. После разделения переменных мы от двумерной спектральной задачи переходим к некоторому числу одномерных задач на собственные значения, каждая из которых приводится к следующему виду:

$$
\begin{gathered}
\psi^{\prime \prime}(x)+L^{2}(U(x)-c) \psi(x)=0, \quad x \in[0, a], \\
\psi(a)=e^{2 \pi i \alpha} \psi(0), \quad \psi^{\prime}(a)=e^{2 \pi i \alpha} \psi^{\prime}(0), \quad 0 \leqslant \alpha<1 .
\end{gathered}
$$

Это индефинитная задача Штурма-Лиувилля, к тому же зависящая от параметра $c$, принадлежащего некоторому конечному промежутку $I$. Пусть $L_{0}(c), L_{1}(c), \ldots$ - собственные значения задачи при фиксированном значении $c$, а $\widetilde{L}_{0}(c), \widetilde{L}_{1}(c), \ldots-B K D$-спектр задачи, т.е. приближенные собственные значения, полученные с помощью ВКБ-приближения.

TEOPEMa 2. 1) $\left|L_{n}(c)-\widetilde{L}_{n}(c)\right| \leqslant$ Const $\left._{1}, \quad 2\right)\left|L_{n}(c)-\widetilde{L}_{n}(c)\right| \leqslant \frac{\text { Const }_{2}}{d^{\delta} \widetilde{L}_{n}(c)}$. 3деcb $d-$ расстояние от с до ближайшего критического уровня функции $U, \delta=7 / 4$. Постояннье Const $_{1} u$ Const $_{2}$ не зависят от параметра $c \in I$.

Автор благодарен А. М. Стёпину за постановку задачи и многочисленные обсуждения. Автор благодарен Р. О. Гриниву за консульации в области спектральной теории.

\section{СПИСОК ЛИТЕРАТУРЫ}

[1] Колокольцов В.Н. // Изв. АН СССР. Сер. матем. 1982. Т. 46. № 5. С. 994-1010. [2] Бабенко И.К., Нехорошев Н. Н. // Матем. заметки. 1995. Т. 58. № 5. С. 643-652. [3] Косыгин Д. В., Минасов А. А., Синай Я. Г. // УМН. 1993. Т. 48. № 4. С. 3-130.

Московский государственньй Принято редколлегией университет им. М.В. Ломоносова 